\title{
Measurement of droplets size and temperature under different conditions by using rainbow technique
}

\author{
Zhaojing $\mathrm{Ni}^{1}{ }^{12}$, Camille Hespel ${ }^{2}$, Fabrice Foucher², Kai Han*1 \\ 1 School of Mechanical Engineering, Beijing Institute of Technology, Beijing, China \\ 2 PRISME, Université d'Orléans, Orléans, France \\ ${ }^{*}$ Corresponding author email: fabrice.foucher@univ-orleans.fr/ autosim@bit.edu.cn
}

\begin{abstract}
The precise measurement of particle size and temperature during the droplet evaporation process is critical yet remains challenging. A non-intrusive method named the rainbow technique is utilized to investigate the evaporation process of ethanol droplets. By analyzing the rainbow patterns captured in the vicinity of the rainbow angle, the average temperature and size of droplet can be simultaneously extracted. Then, the evaporation process of ethanol droplet stream is conducted under ambient temperatures of $293 \mathrm{~K}$ to $313 \mathrm{~K}$. The results validate the accuracy of the rainbow technique in measuring the droplet size and refractive index. Furthermore, a higher ambient temperature and a smaller droplet induce a larger reduction rate in droplet diameter. Moreover, the experimental results are quantitatively compared with the simulation data. Both the numerical and experimental results exhibit a reduction tendency of temperature, revealing the cooling effect as a result of vaporization. Besides, it indicates that the measured temperature by the rainbow technique is the equivalent temperature in the liquid phase.
\end{abstract}

Keywords: Rainbow technique; Droplet size; Droplet temperature; Model validation;

\section{Introduction}

Droplet evaporation plays a central role in many practical domains including engines, spray drying, inkjet printing, spray coating, agricultural irrigation, and so on [1]. Regarding this, the precise measurement of droplet evaporation process is essential in comprehending the mechanism of vaporization phenomenon.

A host of experimental researches have been made on droplet evaporation up to now. The experimental configurations generally fall into two categories: suspended droplets and moving droplets. Although suspension droplets with filament-hanging [2] or acoustic/optical/electrical approaches [3] are prevalent and can be easily operated at elevated temperatures and pressures, it has several distinct drawbacks such as larger droplets (up to millimeter scale), external heat conduction, and over-deviation from practice situation [4]. Concerning this, the investigation of a moving droplet stream can be regarded as an improvement towards the actual situation.

Although the tracking and capturing of moving droplets remains challenging, the optical diagnostic provides a variety of possibilities to evaluate the droplet temperature and size, such as laser-induced fluorescence (LIF), Phase Doppler anemometry (PDA), morphologydependent resonances (MDRs), and rainbow refractometry [5]. Among them, rainbow refractometry has drawn great attention since it is a truly non-intrusive temperature measurement method without any addition of fluorescence product. Without being exhaustive listed, some of the significant contributions have been made by the group of $\mathrm{Wu}$ [6], Saengkaew et al. [7], and Promvongsa et al. [8]. This technique has a wide range of application configurations, such as single droplets, mono-dispersed droplets as well as spray droplets [9]. By measuring the scattering light patterns by droplets nearby the rainbow angle, one can 
obtain the size and refractive index information simultaneously and accurately. Accordingly, it has been a promising optical method applied in droplet measurement.

Hence, we intend to characterize the evaporation process of droplet stream by applying the rainbow technique. As a preliminary work, the transient evaporation of free-falling ethanol droplets under ambient temperatures of $293-313 \mathrm{~K}$ is investigated in the frame of this study. The feasibility of the measurement method is checked and analyzed. Finally, the experimental statistics are quantitatively compared with the simulation data to examine the differences over the test results.

\section{Experimental and image processing}

\subsection{Experimental setup}

The experimental system adopted in the present work is shown in Figure 1. It mainly has three sections, i.e. droplet generation, high-temperature and pressure vessel, and the rainbow imaging system. The droplet stream is generated by a piezoelectric, vibrating orifice droplet generator (MTG-01-G1, FMP). One can obtain a well-controlled mono-disperse droplet stream with equal space and size by adjusting the frequency of generator and the injection pressure. In this experiment, the frequency and injection pressure is equal to $28.73 \mathrm{kHz}$ and $1.2 \mathrm{bar}$, respectively for three pinhole size of 50,75 and $100 \mu \mathrm{m}$. With those operating conditions, the velocity of droplets is approximately $6.8 \mathrm{~m} / \mathrm{s}$ while the diameters of droplets vary with the pinhole size. The temperature of the vessel can be regulated to a maximum value of $473 \mathrm{~K}$ with an accuracy of $\pm 0.1 \mathrm{~K}$. The rainbow imaging system is mainly composed of two parts, emitting part and receiving part. A vertically polarized $532 \mathrm{~nm}$ pulsed laser beam (repetition rate $20 \mathrm{~Hz}$ ) with a size of around $2 \mathrm{~mm}$ from Thales illuminates the droplet stream. The output energy is $10 \mathrm{~mJ}$ with a duration of $7.5 \mathrm{~ns}$. The scattered light from droplets is then collected by the Plano-convex lens (with $150 \mathrm{~mm}$ focal length and $75 \mathrm{~mm}$ diameter) at a distance of 278 $\mathrm{mm}$. Then the image is received by the CCD camera $(2048 \times 2048$ pixels, $14.6 \mu \mathrm{m} /$ pixel). The measurement volume spans the vertical distance $35-42 \mathrm{~mm}$ away from the nozzle of droplet generator.

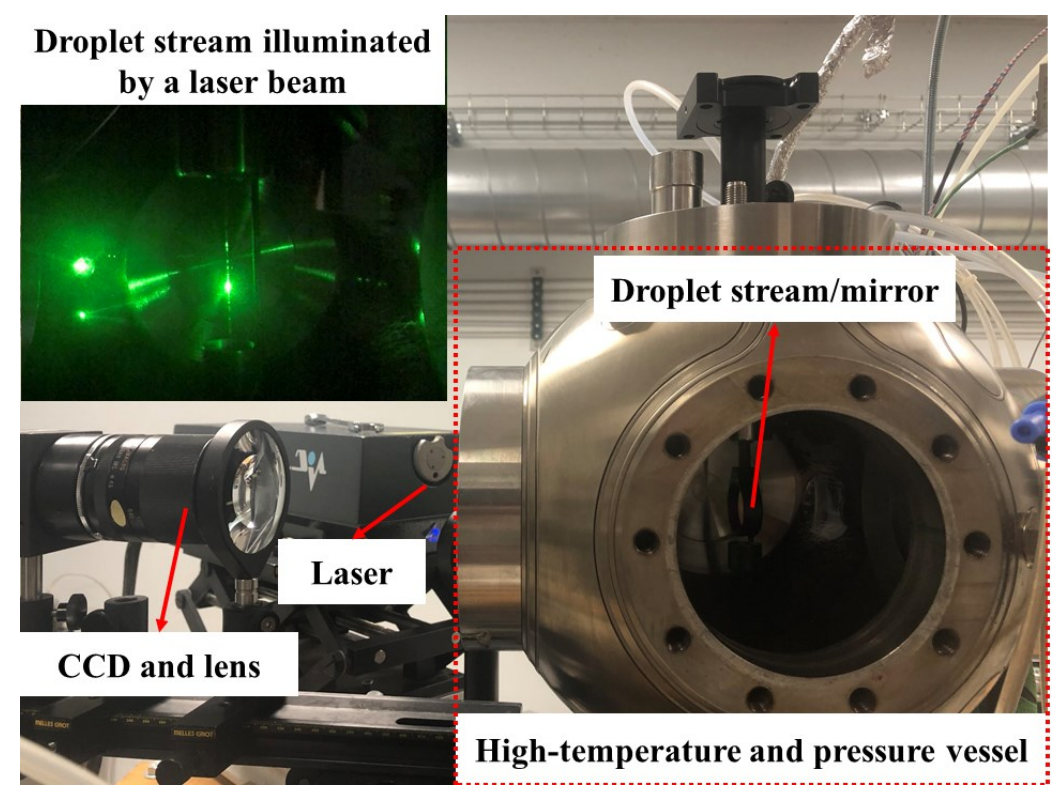

Figure 1. Composition of the experimental setup.

\subsection{Typical scattering light patterns by experiment}


Figure 2 shows a typical rainbow image and corresponding intensity curve of the droplet stream. Those patterns come from the interference between first internal reflections. To make quantitative analysis, the scattering diagrams (intensity versus pixels) can be extracted from the recorded image by averaging a given number of 20 horizontal lines over the central region of the pattern, as indicated in Figure $\mathbf{2} \mathbf{b}$. This process aims to reduce the high-frequency noise of light patterns and get a relatively smooth curve. As indicated, the main features of the primary rainbow are clearly displayed. For the primary rainbow, both the main rainbow peak and Airy fringes are observed. In addition, the high-frequency fringes named 'ripple structures' superimposing on the Airy fringes are also visible, which validates the stability of droplet stream. Those fringes result from the interference between the external reflection and internal reflection light [10]. For the typical scattering light patterns, the rainbow position can be used to determine the droplet temperature while the rainbow shape changes with the droplet size. Therefore, the most important two parameters are the primary rainbow angle and the distance of airy fringes [11].

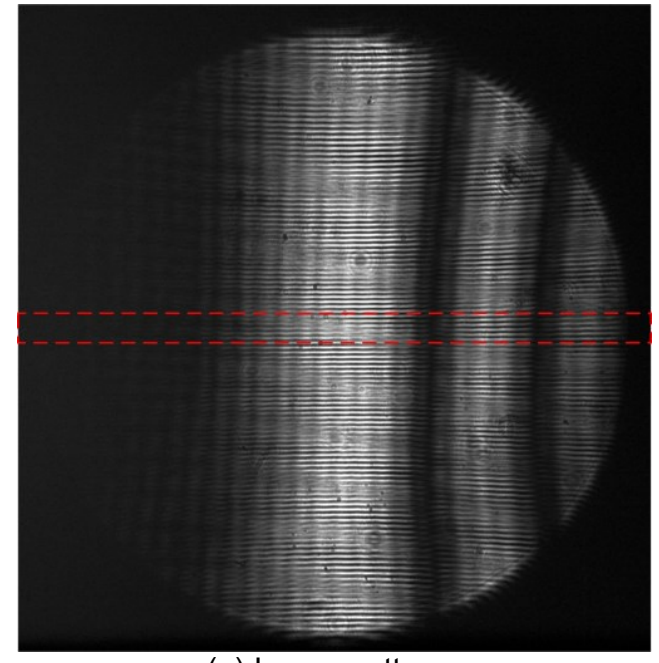

(a) Image patterns

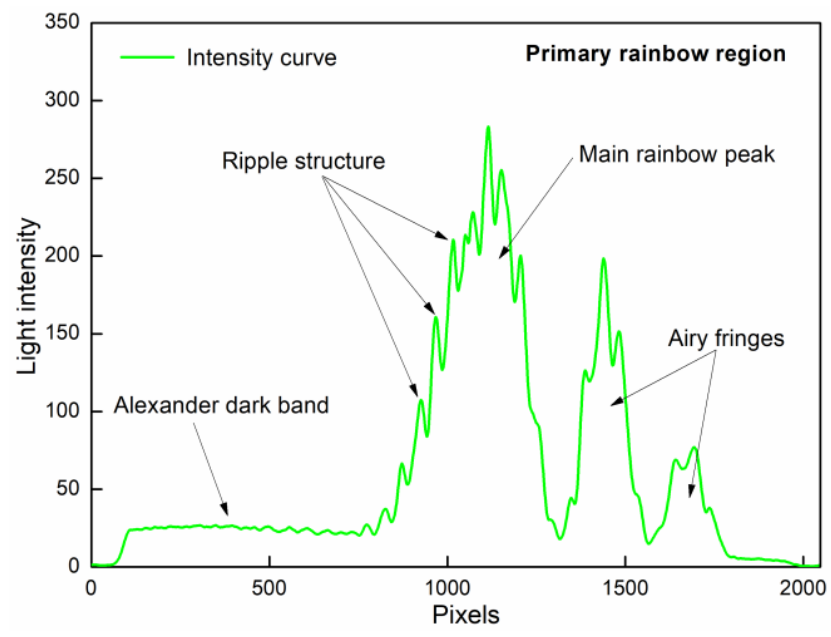

(b) Intensity curve versus pixels

Figure 2. Typical rainbow signal and intensity curve; (a) Rainbow signal of ethanol droplets illuminated by a pulsed laser at the atmospheric pressure and temperature condition (pinhole size of $100 \mu \mathrm{m}$, frequency 28.97 $\mathrm{kHz}$, injection pressure of 1.2 bar); (b) The intensity curve averaged over 20 lines along the center.

\subsection{Angular calibration}

The angular-pixel calibration aims to relate the pixels to angle for rainbow signal processing. The calibration procedures are conducted by placing a mirror exactly at the probe line segment mounted on the center of a precision rotation platform. The laser beam is reflected by the mirror, perpendicularly incidents on the first lens and propagates to the CCD as the recorded rainbow light does. The image position on the CCD sensor of the laser beam with different incident angles is obtained by rotating the mirror. Figure $\mathbf{3 a}$ displays the reflected light intensity curves of the laser beam at five different angles. By finding the center of the plateau in the intensity curve, the relationship between angles and pixels can be retrieved. As shown in Figure $\mathbf{3 b}$, a good linearity between the scattering angle and the pixel is observed and calculated. A tiny change of three decimals of scattering angle values can be detected by the present camera resolution of $2048 \times 2048$. Noting that the angular calibration is necessary after each experiment to avoid the inaccuracy caused by any tiny changes. 


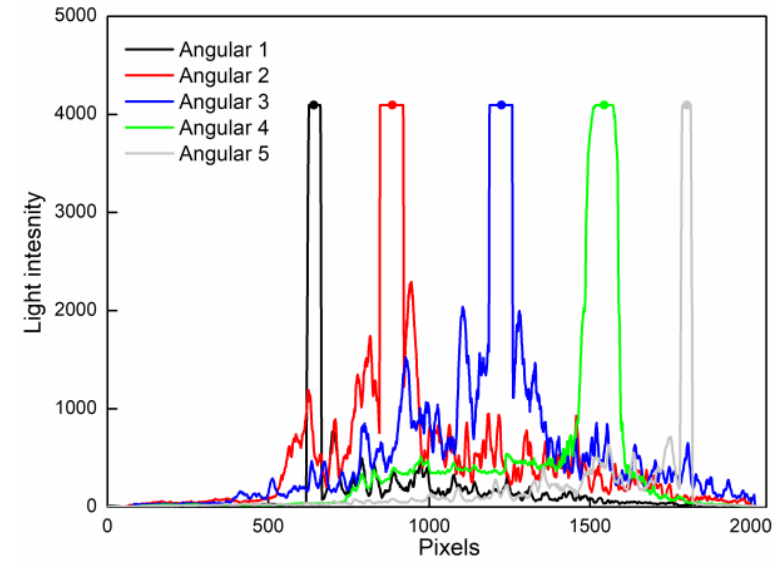

(a) The light intensity curve at different angles

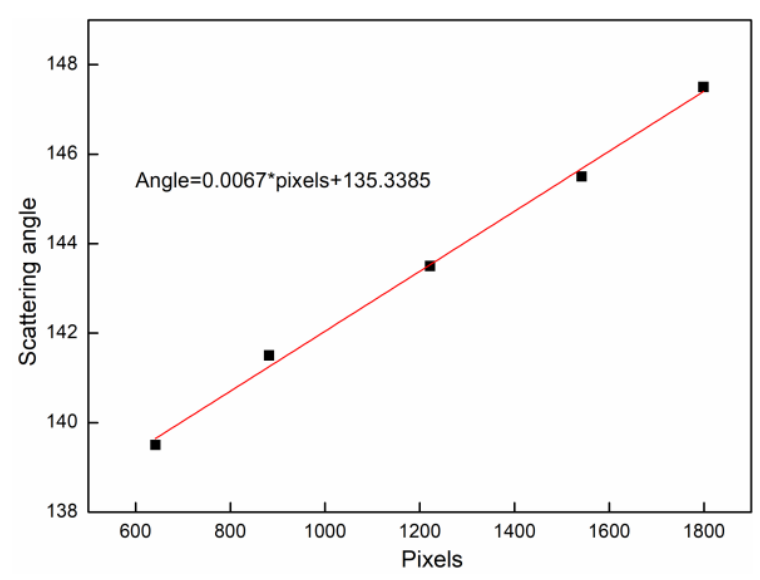

(b) The calibrated angle-pixels relation

Figure 3. Typical rainbow calibration process; (a) The reflected light intensity curve of laser beam at different angles; (b) The calibrated angle-pixels relation.

\subsection{Retrieve of diameter and refractive index}

After a series of pre-processing operations, a smooth intensity curve without ripple structure is obtained. Then, the filtered curve is chosen as reference data, from which the angular position of first and second peak values $\theta_{1}$ and $\theta_{2}$ are determined. The simulation method utilized in the retrieve process is mainly done by Airy theory, which is efficient and effective in predicting the rainbow patterns near the rainbow angle as demonstrated in previous researches [12]. According to the Airy theory, the relation between the rainbow angle $\theta_{r g}, \theta_{1}$ and $\theta_{2}$ is given by [13]:

$$
\theta_{r g}=\left(\theta_{1}-c \theta_{2}\right) /(1-c)
$$

where $c$ is a constant defined as $c=\alpha_{1} / \alpha_{2}$ with $\alpha_{1}=1.0874, \alpha_{2}=3.4668$. After knowing the rainbow angle, we can implicitly calculate the relative refractive index by the following relation:

$$
\theta_{r g}=\pi+2 \cos ^{-1}\left(\sqrt{\frac{N^{2}-1}{p^{2}-1}}\right)-4 \cos ^{-1}\left(\sqrt{\frac{p^{2}\left(N^{2}-1\right)}{\left(p^{2}-1\right) N^{2}}}\right)
$$

where $N$ is the refractive index and $p=2$. After ensuring the refractive index, the particle diameter is predicted by iteratively simulating a bunch of candidate intensity light patterns with the Airy theory. The least-squares method is used to pick the optimum candidate curve with reference data. Apart from this, the other two diameter values named airy diameter $D_{\text {airy }}$ and ripple diameter $D_{\text {ripple }}$ could also be calculated from the experimental data by the following formulas [14]:

$$
\begin{aligned}
& D_{\text {airy }}=\frac{\lambda}{4}\left[\left(\frac{\left(p^{2}-1\right) \sqrt{\left(p^{2}-N^{2}\right)}}{\left(N^{2}-1\right)^{3 / 2}}\right)^{1 / 2}\left(\frac{\alpha_{1}-\alpha_{2}}{\theta_{1}-\theta_{2}}\right)^{3 / 2}\right] \\
& F_{\text {ripple }}=\frac{D_{\text {ripple }}}{2 \lambda}\left(\cos \tau_{r g}+\cos \frac{\theta_{r g}}{2}\right) \frac{\pi}{180^{\circ}}
\end{aligned}
$$

Where $F_{\text {ripple }}$ is the ripple frequency, which can be judged from the angular frequency spectrum of scattering light patterns. The diameter values predicted by the two methods provide a good avenue to ensure the accuracy of the object diameter by iteration. 


\section{Results and discussion}

\subsection{Reliability and repeatability check}

To verify the stability of droplet generation as well as the reliability of the measurement method, droplets at different distances from the nozzle ranging from 35-42 $\mathrm{mm}$ are measured under room temperature and pressure conditions. A stream of ethanol droplets is generated with a pinhole size of $100 \mu \mathrm{m}$. In the first, the diameter evaluated by iteration, ripple frequency, and airy peaks is compared in Figure 4. Theoretically, the particle size predicted by those three methods should be uniform with each other [15]. However, as expected, the droplet diameter evaluated by three methods differs slightly when it comes to the experimental situation. Despite that, the ripple diameter and airy diameter offer a good reference for the iteration diameter. The relative difference of ripple and airy diameter against the iteration diameter is also checked with no more than $3 \%$ of errors. This ensures the accuracy of diameter measurement by the present post-processing process.

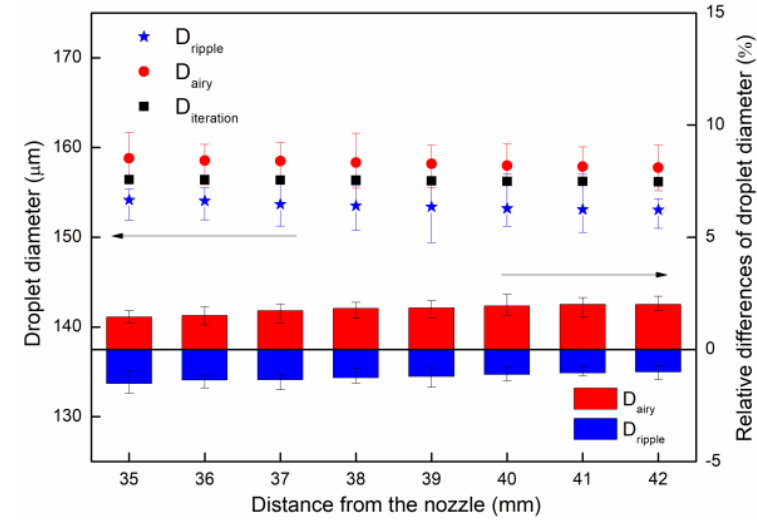

(a) Reliability check

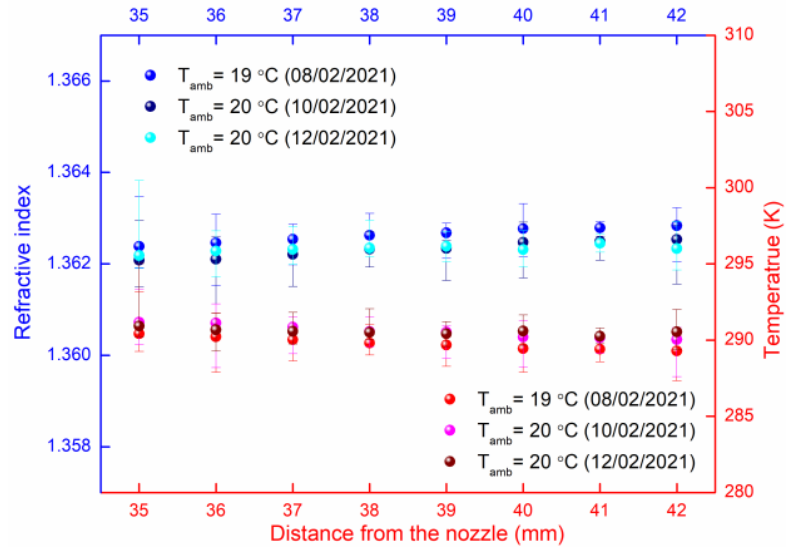

(b) Repeatability check

Figure 4 . The reliability and repeatability examination of the measurement method.

The repeatability of the measurement method is checked by conducting the experiment on three different days. The refractive index together with the temperature are compared in Figure 4b. the equation provided by Smith et al. [16] is used for evaluating the temperature from the refractive index:

$$
N=1.36929-0.000400 T
$$

The measured temperature value is lower than the actual room temperature on that day, which can be attributed to the convective evaporation taking away heat from the droplet and cooling the liquid phase. It can be concluded that the present rainbow measurement method is reproducible and reliable. In the following research, 100 scattering images are sampled at least under each condition to ensure the reliability of experimental data.

\subsection{Influence of the ambient temperature and droplet diameter}

The variation of droplet diameter and temperature at different temperature conditions are compared in Figure 5. The initial velocity from the nozzle is about $6.8 \mathrm{~m} / \mathrm{s}$, accordingly, the velocity along the measured volume is $6.85-6.86 \mathrm{~m} / \mathrm{s}$ due to the gravity acceleration. Since the control volume spans 35-42 $\mathrm{mm}$ away from the nozzle of droplet generator, the distance information turns into a time range of 0.00513-0.00615 s. As displayed in Figure 5, although 
the variation of diameter is limited by the small control volume and the low temperatures, the droplet diameter gradually goes down under different ambient temperature conditions revealing the existence of evaporation. Besides, the reduction of droplet diameter is accelerated by promoting the ambient temperature, which is agreeable with general cognition. Additionally, the variation of temperature is inversely proportional to the refractive index. $A$ small refractive index means a high temperature and vice versa. A cooling process in droplet temperature is observed, which could be mitigated by enhancing ambient temperature.

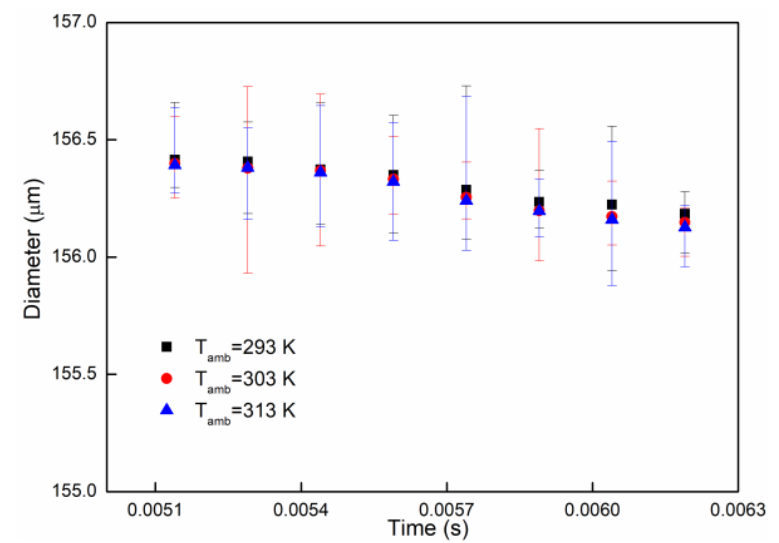

(a) Droplet diameter

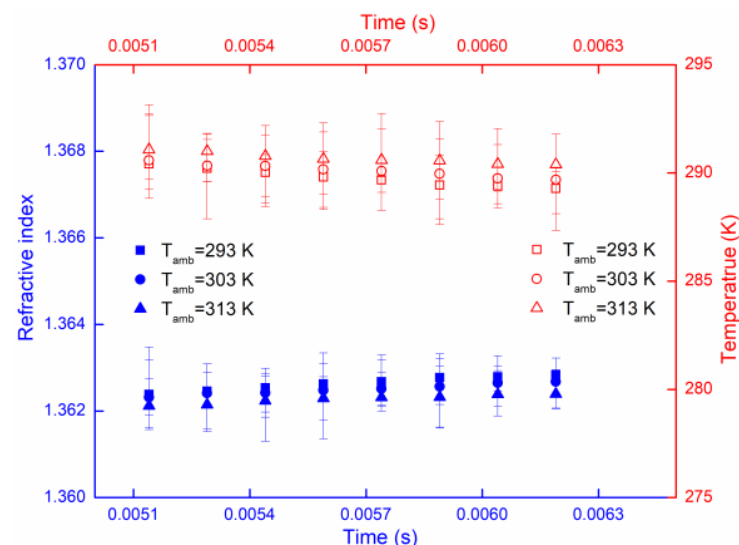

(b) The refractive indices and temperatures

Figure 5. The droplet diameter, refractive indices, and temperatures at different temperatures.

Similarly, the variation of droplet diameter and temperature by using three different pinhole sizes of 50.75, and $100 \mu \mathrm{m}$ are displayed in Figure 6. The measurements were conducted at an ambient temperature of $313 \mathrm{~K}$ and normal pressure conditions. First of all, a reduction trend of droplet size with time is observed for different droplet streams. The evaporation of smaller droplets is more intense than large droplets in the present research range. The droplet diameter generated with pinhole size of $50 \mu \mathrm{m}$ reduces by $0.72 \%$ while leaving the control volume compared with the time when it entered the measured volume. By contrast, the reduction rate in diameter is almost invisible with a value of $0.17 \%$ for the pinhole size 100 $\mu \mathrm{m}$. On the other hand, a larger reduction rate in diameter means that vaporization dominates much of the energy transferred into the droplet, which impedes the heating process in the liquid phase. Consequently, smaller droplets always maintain a lower temperature in the liquid phase compared with larger droplets as shown in Figure 6b.

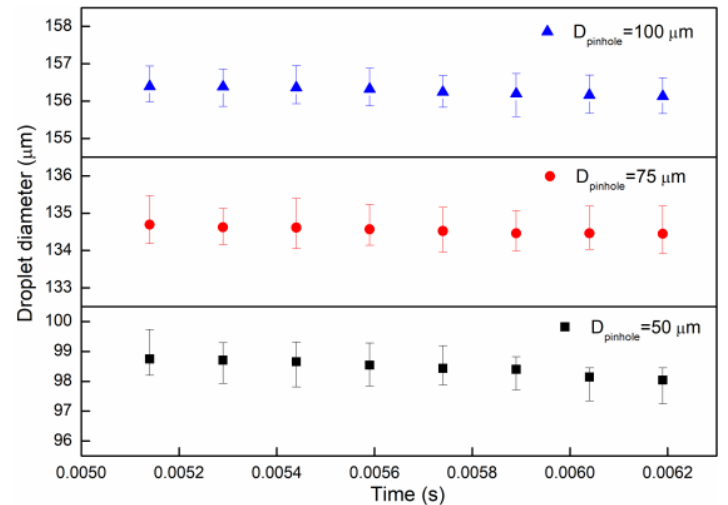

(a) Diameter

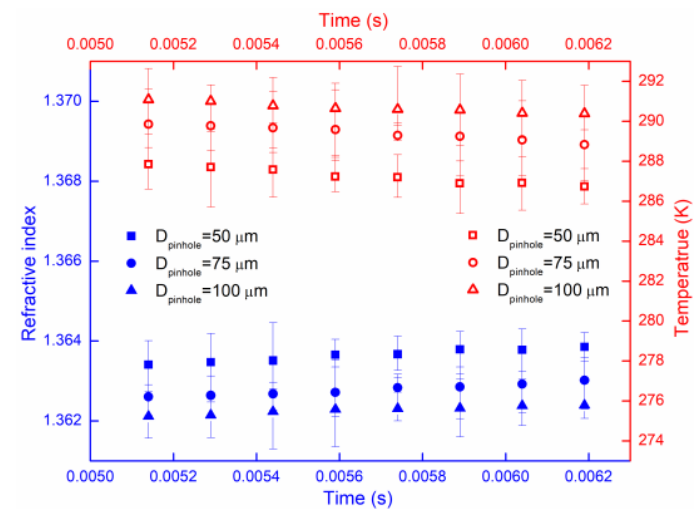

(b) Refractive indices and temperature

Figure 6. Variation of the droplet diameter, refractive indices, and the corresponding temperatures generated by different pinhole sizes. 


\subsection{Comparison between experimental and simulation results}

In this section, the measured data are compared with simulation results to ensure the accuracy of the experimental method as well as providing a further benchmark for model validation. The vaporization model proposed in the authors' previous work is used to mimic the evaporation process [17, 18]. As shown in Figure 7, an ethanol droplet stream generated from the pinhole size of $100 \mu \mathrm{m}$ vaporizes at three ambient temperature conditions. The simulation adopted the same boundary and initial conditions with the experiment, i.e. the initial droplet temperature $293 \mathrm{~K}$ and a convective velocity of $6.85 \mathrm{~m} / \mathrm{s}$. The initial droplet diameter of $157.5 \mu \mathrm{m}$ has been chosen to have a better comparison with simulation data. For the present research range, although a diameter reduction is observed for all three cases, the difference of droplet diameter among individual cases is almost invisible due to the restriction of a relatively lower ambient temperature and the measurement volume. However, this behavior confirms well with the simulation results during this early stage of evaporation.

In addition, the temperature variations are displayed in Figure $\mathbf{7 b}$. Firstly, for simulation, the temperatures at the droplet surface are always lower than the ones at the droplet center since it is more sensitive to the change in the gas phase. Besides, both the numerical and experimental results exhibit a reduction tendency of temperature, revealing the cooling effect as a result of vaporization. Furthermore, the measured droplet temperatures are between the predicted values at the droplet surface and center, with a more approximation to the temperature at the droplet center. This could be attributed to the temperature gradient inside the droplet, which changes the light path over the droplet center. Therefore, the measured temperature by the rainbow technique can be regarded as the equivalent temperature in the liquid phase and is more close to the temperature value at the droplet center.

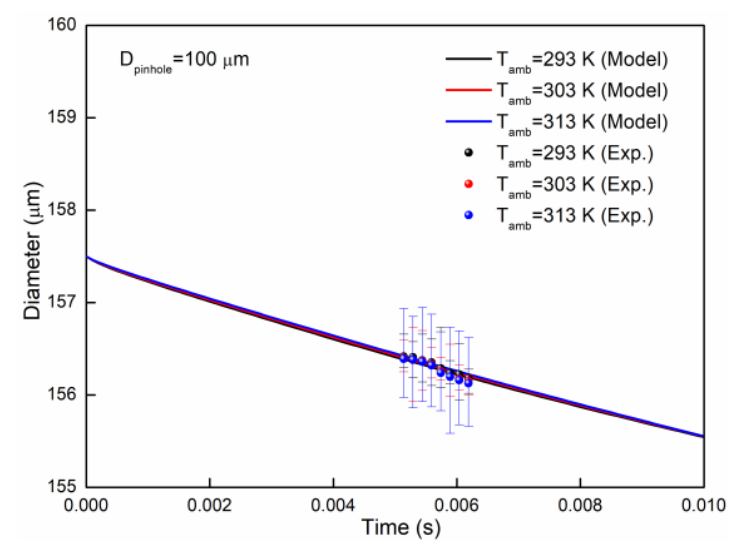

(a) Diameter

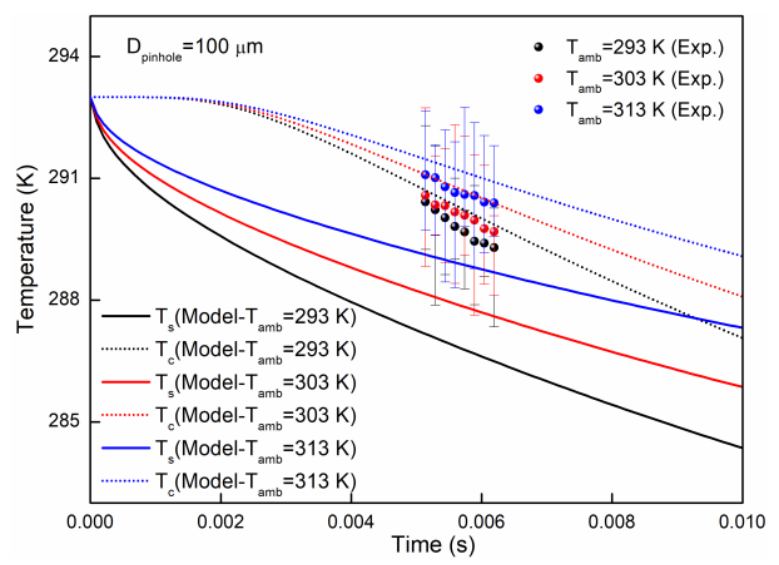

(b) Temperature

Figure 7. Variation of the droplet diameter, refractive indices and the corresponding temperatures generated by different pinhole sizes.

\section{Concluding remarks}

This study experimentally investigated the evaporation process of ethanol droplet streams by establishing the rainbow imaging setup. The experiment was conducted under ambient temperatures of $293 \mathrm{~K}$ to $313 \mathrm{~K}$. The results demonstrated the accuracy of the rainbow technique in measuring the droplet size and refractive index. Furthermore, the droplet diameter gradually goes down under different ambient temperature conditions revealing the existence of evaporation. The reduction of droplet diameter is accelerated by promoting the ambient temperature. Moreover, the evaporation of smaller droplets is more intense than large 
droplets in the present research range, which in turn impedes the heating process in the liquid phase. As a result, smaller droplets always maintain a lower temperature in the liquid phase compared with larger droplets. Finally, the experimental results are quantitatively compared with the simulation data. Both the numerical and experimental results exhibit a reduction tendency of temperature, revealing the cooling effect as a result of vaporization. Besides, it indicates that the measured temperature by the rainbow technique is the equivalent temperature in the liquid phase and is more close to the temperature value at the droplet center due to the temperature gradient inside the droplet.

\section{Acknowledgments}

The financial support provided by the China Scholarship Council (No.201806030456) for a research visit of the lead author to the Université d'Orléans is acknowledged. Also the experiments were conducted at the lab of PRISME, Orleans.

\section{References}

[1] Li, C., Lv, Q., Wu, Y., Wu, X., and Tropea, C., 2020, International Journal of Heat and Mass Transfer, 146, pp. 118843.

[2] Abou Al-Sood, M. M., and Birouk, M., 2008, International Journal of Heat and Mass Transfer, 51(5-6), pp. 13131324.

[3] Al Zaitone, B., 2018, International Journal of Heat and Mass Transfer, 126, pp. 164-172.

[4] Wang, J., Huang, X., Qiao, X., Ju, D., and Sun, C., 2020, Fuel, 268, pp. 117407.

[5] Perrin, L., Castanet, G., and Lemoine, F., 2015, Experiments in Fluids, 56(2), pp. 1-16.

[6] Wu, Y., Crua, C., Li, H., Saengkaew, S., Mädler, L., Wu, X., and Gréhan, G., 2018, Journal of Quantitative Spectroscopy and Radiative Transfer, 214, pp. 146-157.

[7] Saengkaew, S., Allano, D., Brunel, M., and Grehan, G., 2014, Optics Communications, 332(332), pp. $269-278$.

[8] Promvongsa, J., Fungtammasan, B., Gerard, G., Saengkaew, S., and Vallikul, P., 2017, Journal of Energy Resources Technology, 139(6), pp. 062002.

[9] Van Beeck, J., Giannoulis, D., Zimmer, L., and Riethmuller, M., 1999, Optics Letters, 24(23), pp. 1696-1698.

[10] Saengkaew, S., Charinpanikul, T., Laurent, C., Biscos, Y., Lavergne, G., Gouesbet, G., and Gréhan, G., 2010, Experiments in Fluids, 48(1), pp. 111-119.

[11] Wilms, J., and Weigand, B., 2007, Applied Optics, 46(11), pp. 2109-2118.

[12] Saengkaew, S., Charinpanitkul, T., Vanisri, H., Tanthapanichakoon, W., Mees, L., Gouesbet, G., and Grehan, G., 2006, Optics Communications, 259(1), pp. 7-13.

[13] Hulst, H. C., and van de Hulst, H. C., 1981, Light scattering by small particles, Courier Corporation.

[14] van Beeck, J. P., and Riethmuller, M. L., 1997, Particle \& particle systems characterization, 14(4), pp. 186192.

[15] van Beeck, J. P. A. J., 1997, PhDT, pp. 150.

[16] Smith, T., and Bonner, R., 1952, Analytical Chemistry, 24(3), pp. 517-518.

[17] Ni, Z., Hespel, C., Han, K., and Foucher, F., 2021, International Journal of Heat and Mass Transfer, 164, pp. 120401.

[18] Ni, Z., Han, K., Zhao, C., Chen, H., and Pang, B., 2018, Fuel, 230, pp. 27-36. 